\title{
A Fully Automatic Algorithm for the Analysis of Heart Rate Changes and Cardiac Recovery during Exercise
}

\author{
M Vaglio ${ }^{1}$, A Porta $^{2}$, P Pizzinelli $^{3}, \mathrm{~S}_{\text {Di Marco }}{ }^{3}$, \\ D Lucini ${ }^{3}$, F Badilini $^{1}$, M Pagani $^{3}$ \\ ${ }^{1}$ AMPS 1lc, New York, NY, USA \\ ${ }^{2}$ Department of Technologies for Health, Galeazzi Orthopaedic Institute, University of Milan, Italy \\ ${ }^{3}$ CTNV, Sacco Hospital, Milan, Italy
}

\begin{abstract}
Exercise test is a challenging experimental condition for signal processing tools since stationarity requirement is not fulfilled.

The aim of the present study was to develop a new way to look at HR and RR variability in exercise test, focusing both on exercise and the subsequent recovery phase.

From RR series, the first derivative of the filtered tachogram and the variance were calculated in windows of predefined length and the three experimental conditions (rest, exercise and recovery) were automatically detected.

Results indicate that in healthy subjects variance is larger during recovery than during exercise for a given $R R$ interval, this is not seen in heart failure patients. In healthy subjects tonic and dynamical control of heart rate should be regarded as different autonomic regulations.
\end{abstract}

\section{Introduction}

The Post-exercise heart rate (HR) recovery and HR variability are commonly used non-invasive assessment procedures for determination of cardiovascular autonomic function.

A delay in HR recovery has been observed in patients with chronic heart failure

Studies of the electrocardiogram during and after exercise (walking on a treadmill ergometer at standard speed and grade) in normal subjects have been performed for very long time $[1,2]$.

Several techniques have been used varying from the analysis of heart rate variability [3] prior and after exercise, Poincaré [4] plots to the analysis of heart rate (HR) sudden increase [5, 6] and other HR changes [7] with exercise and HR recovery after exercise [8-10].

Normal subjects as well as several pathologies have been subjected to exercise test

In the field of sport testing, heart rate variability analysis has been used to evaluate modifications of autonomic cardiovascular functions during exercise or after a training period.

Exercise test is a challenging experimental condition for signal processing since it is a non static process.

In this study we present a new way to look at exercise testing where information on HR recovery and HR variability changes are presented simultaneously.

\section{Methods}

\section{Study Population}

Our study includes 10 heart failure (CHF) patients, five of which were females and 12 healthy controls (C).

All subject attended an initial rest-test that was used for validating the healthy/heart failure condition.

On the day of the recording, subjects were recorded at standing resting position for almost five minutes, then in walking condition for an additional 3 minutes. At this point the exercise submaximal test, following a Bruce protocol, took place. Finally, within $30 \mathrm{~s}$ form the end of the exercise phase, subjects were positioned in supine position, to start the recovery phase, which was approximately 5 minutes long.

\section{Algorithm description}

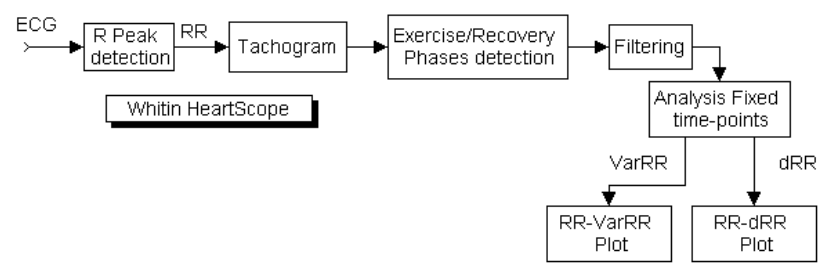

Figure 1. Block diagram of the various signal processing steps undertaken in the study. Detailed description is offered in Methods chapter.

ECGs were recorded during exercise with MP150 data acquisition systems (BIOPAC Systems Inc, Goleta, CA). From the recorded ECGs, RR series were automatically extracted within HeartScope (AMPS llc, New York, NY) 
[11], software that allows the analysis of cardiovascular signals, from the lead that presented tallest R-waves.

From RR series, the three experimental conditions (rest, exercise and recovery) were automatically detected.

The tachogram was then filtered by a MA filter with a window size of 20 subsequent $R R s\left(F_{R R}\right)$. Afterwards $F_{R R}$ was once more filtered with a derivative filter to obtain $\mathrm{dF}_{\mathrm{RR}}$.

The following step was averaging $\mathrm{dF}_{\mathrm{RR}}$ series at fixed time points (4s resolution) with a MA filter and the computation of the variance of predefined windows at the same fixed time points of the tachogram to obtain $\operatorname{Var}_{\text {RR }}$.

For comparison purpose, $R R$ and both $\mathrm{dF}_{\mathrm{RR}}$ and $\operatorname{Var}_{\mathrm{RR}}$ were normalized $\left(\operatorname{Var}_{R R n}\right.$ and $\left.\mathrm{dF}_{\mathrm{RRn}}\right)$ using maximum and minimum values.

The final step of the algorithm is the quantification of the area in the phase plane (RR - VarRR) and (RR dRR).

For statistic analysis, nonparametric Kruskal-Wallis test on median was used, p-value $<0.05 \quad\left(^{*}\right)$ was considered significant level, $p$-value $<0.1$ (†) was also marked.

\section{Results}

Both exercise and recovery phases present large differences in the two studied populations.

Automatically extracted exercise phases were $398 \pm 180$ and $431 \pm 252$ seconds long, while recovery phases were $193 \pm 78$ and $160 \pm 61$ seconds long for C and CHF respectively.

As reported in Table 1, time-domain parameter such as maximum HR at exercise peak was significantly higher in healthy individuals, so for $\Delta \mathrm{RR}$ at $80 \mathrm{~s}$ into recovery phase: HR recovery is almost twofold for healthy, although significance is not reached.

Our new developed parameters on phase-plane analysis present markedly higher values for healthy subjects, precisely VarRR during the recovery phase (after $40 \mathrm{~s}$ from beginning of recovery) was almost two fold in $\mathrm{C}$ than CHF.

The observation of Fig 2 and 3 gives an overview of the analysis on phase-plots. For healthy subjects, the variance in the recovery phase is much higher than the exercise phase or rest state.

The trajectories on the phase-plot in heart failure patients were totally perturbed, the monotonic behavior of the initial phase of the recovery was modified to an oscillating pattern.
Table 1. Several analyzed parameters for the two studied population. Results are given as mean \pm std. KruskalWallis test: $\left(^{*}\right)$ p-value $<0.05,(\dagger) p$-value $<0.1$.

\begin{tabular}{|l|l|l|c|}
\hline & CHF $(\mathrm{n}=10)$ & $\mathrm{H}(\mathrm{n}=12)$ & $\mathrm{p}$-value \\
\hline Age & $67 \pm 11$ & $39 \pm 12$ & $*$ \\
\hline Gender $(\mathrm{F})$ & $50 \%$ & $17 \%$ & $*$ \\
\hline $\begin{array}{l}\mathrm{HR} \text { at peak } \\
\text { Exercise (bpm) }\end{array}$ & $118 \pm 16$ & $172 \pm 17$ & $\dagger$ \\
\hline $\begin{array}{l}\Delta \mathrm{RR} \text { at 80s in } \\
\text { rec. }(\mathrm{ms})\end{array}$ & $110 \pm 57$ & $203 \pm 148$ & $\dagger$ \\
\hline $\begin{array}{l}\mathrm{Var}_{\mathrm{RR}} \text { at 80s in } \\
\text { rec. }\left(\mathrm{ms}^{2}\right)\end{array}$ & $1507 \pm 4203$ & $3106 \pm 5813$ & \\
\hline $\begin{array}{l}\mathrm{dF}_{\mathrm{RR}} \text { at 80s in } \\
\text { rec. }\end{array}$ & $446 \pm 448$ & $1226 \pm 1751$ & $\dagger$ \\
\hline $\mathrm{Var}_{\mathrm{RR}}$ area & $67 \pm 148$ & $110 \pm 218$ & $\dagger$ \\
\hline $\mathrm{dF}_{\mathrm{RR}}$ area & $7.1 \pm 3.2$ & $33.2 \pm 36.7$ & $*$ \\
\hline
\end{tabular}
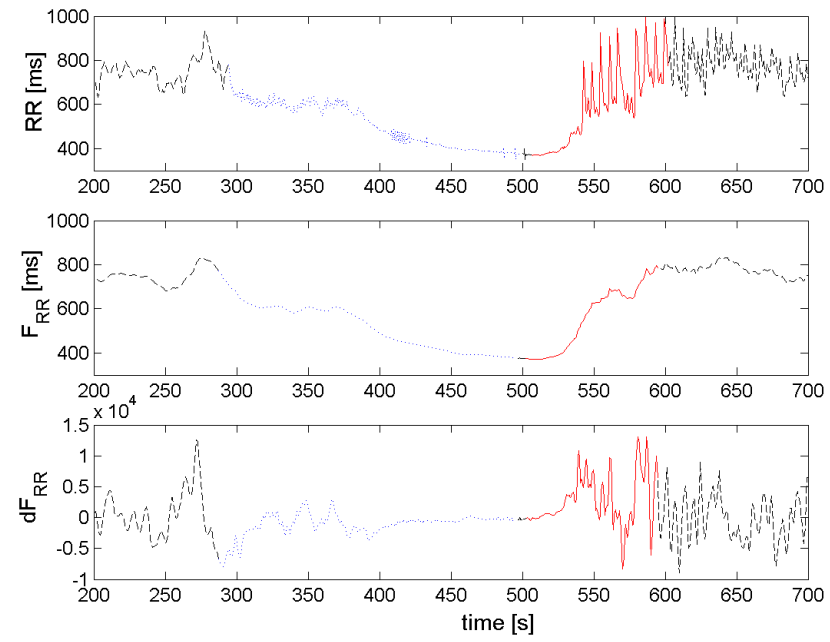

Figure 2. The tachogram, the filtered tachogram and $\mathrm{dF}_{\mathrm{RR}}-\mathrm{RR}$ for an healthy individual are here shown. The two exercise phases are also visualized differently, dotted line for exercise and solid line for recovery phase. A clear reactivation is visible a minute into recovery phase (sudden RR interval increase and appearance of marked $\mathrm{dF}_{\mathrm{RR}}$ oscillations). 

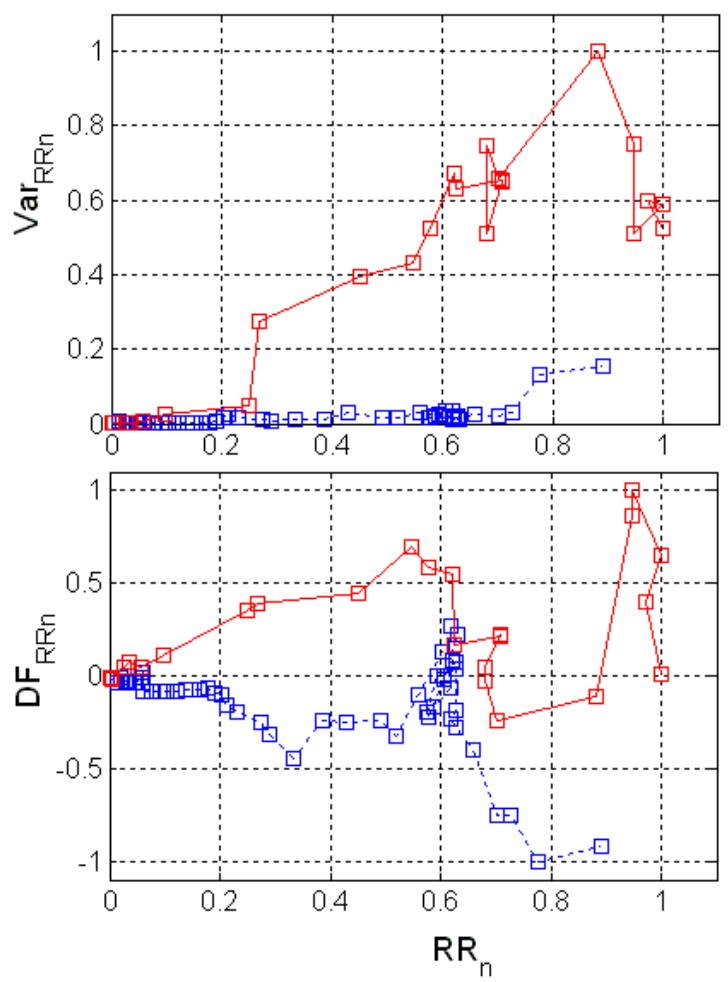

Figure 3. Example of normalized $\operatorname{Var}_{R R}-R R$ (upper) and $\mathrm{d}_{\mathrm{RR}}-\mathrm{RR}$ (lower) plots for an healthy individual. Time points are $4 \mathrm{~s}$ apart. It is clear that during the exercise (dotted line) phase VarRR is seriously reduced, while it quickly increases after 20s from the beginning of the recovery (solid line) phase. $d R R$ indicates a clear RR decrease during late exercise phase and a monotonic RR increase starting with the beginning of recovery.

\section{Discussion and conclusions}

The plots clearly indicate that in healthy subjects at a given RR interval the variance is larger during recovery than during exercise and even during rest, thus producing a sort of loop covered clockwise in the phase plane (RR VarRR) and (RR - dRR). In healthy subjects the trajectory is not completely closed since the final mean $R R$ interval is significantly larger than that during resting condition. This trajectory collapses onto itself in heart failure patients. These results support the conclusions that:

i) in healthy subjects tonic control of heart rate, setting the mean value of RR interval, and dynamical control, setting the level of heart rate variability, should be regarded as different autonomic regulations;

ii) a deeper analysis of the form of the trajectory in the phase plane (RR - VarRR) and (RR - dRR) during exercise test may unveil important details of the dynamics of cardiac autonomic nervous system regulation.

Our results should be regarded as preliminary, since age and fitness levels of the examined populations were not matched.

Accordingly, it will be fundamental to validate our findings on a larger and more homogeneous cohort.

This technique might also be useful for quantifying fitness level, analysing groups of healthy subjects before and after physical training.
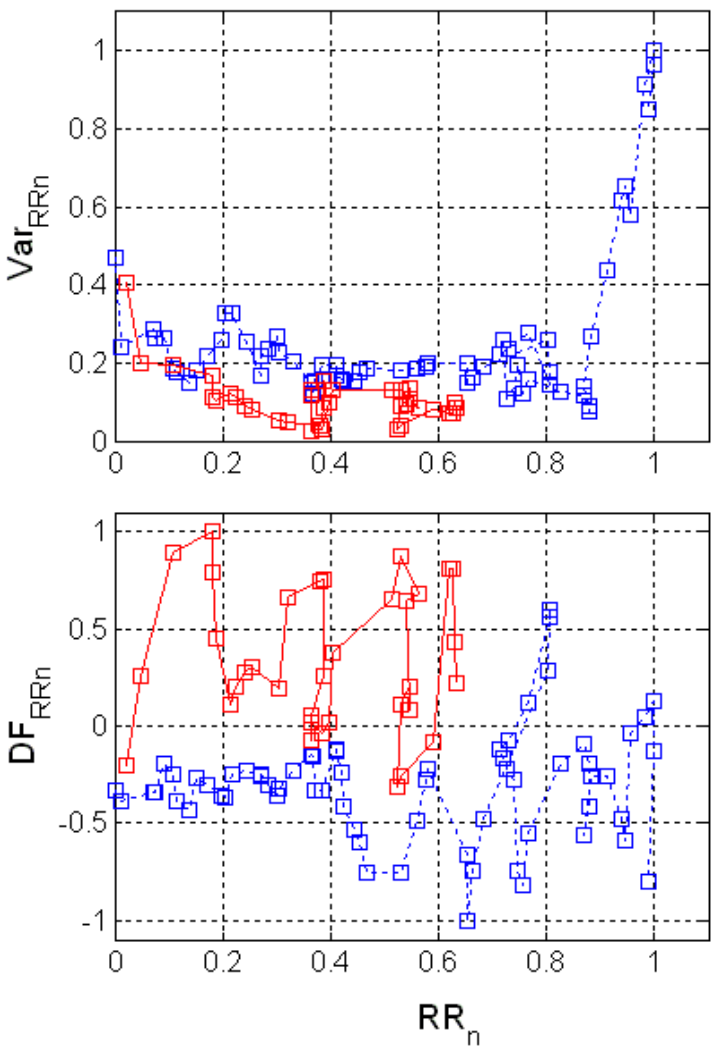

Figure 4. Example of normalized $\operatorname{Var}_{R R}-R R$ and $d_{R R}-R R$ plots for a CHF subject. Time points are $4 \mathrm{~s}$ apart. During exercise (dotted line) phase VarRR is reduced, but during recovery (solid line) phase it remains seriously reduced. The process of autonomic recovery is seriously perturbed. $\mathrm{dF}_{\mathrm{RR}}$ shows an oscillatory behavior.

\section{References}

[1] Missal ME. Exercise tests and the electrocardiogram in the study of angina pectoris. Ann. Int. Med 1938;1:2018.

[2] Yu PNG. Studies of Electrocardiographic Changes during Exercise (Modified DoubleTwo-Step Test). Circulation 1952; 6:183-192.

[3] Iellamo F, Pigozzi F, Spataro A, Lucini D, Pagani M. TWave and Heart Rate Variability Changes to Assess Training in World-Class Athletes. Med Sci Sports Exerc 2004; 36:1342-1346. 
[4] Mourot L, Bouhaddi M, Perrey S, Rouillon JD, Regnard J. Quantitative Poincare' plot analysis of heart rate variability: effect of endurance training. Eur J Appl Physiol 2004; 91:79-87.

[5] Lauer MS, Okin PM, Larson MG, Evans JC, Levy D. Impaired Heart Rate Response to Graded Exercise. Circulation 1996; 93:1520-1526.

[6] Leeper NJ, Dewey FE, Ashley EA, Sandri M, Tan SY, Hadley D, Myers J, Froelicher V. Prognostic Value of Heart Rate Increase at Onset of Exercise Testing. Circulation 2007; 115:468-474.

[7] Krahn AD, Klein GJ, Yee R. Hysteresis of the RT Interval With Exercise. Circulation 1997; 96:1551-1556.

[8] Nissinen SI, Mäkikallio TH, Seppänen T, Tapanainen JM, Salo M , Tulppo MP, Huikuri HV. Heart rate recovery after exercise as a predictor of mortality among survivors of acute myocardial infarction. Am J Cardiol 2003; 91:711-714.

[9] Nanas S, Anastasiou-Nana M, Dimopoulos S, Sakellariou D, Alexopoulos G, Kapsimalakou S, Papazoglou P, Tsolakis E, Papazachou O, Roussos C. Early heart rate recovery after exercise predicts mortality in patients with chronic heart failure. Int J Cardiol 2005; 110:393-400.
[10] Huang PH, Leu HB, Chen JW, Lin SJ. Heart Rate Recovery After Exercise and Endothelial Function-Two Important Factors to Predict Cardiovascular Events. Prev Cardiol 2005;8:167-170.

[11] Badilini F, Pagani M, Porta A. HeartScope: a Software Tool Addressing Autonomic Nervous System Regulation. Computers in Cardiology 2005;32:259-262.

Address for correspondence:

Martino Vaglio

Via Paolo VI, 34

25018 Montichiari (BS) - Italy

Phone: +390309650745

Fax: +390309650572

e-mail : vaglio@amps-llc.com 